\title{
Serial Angioscopic Evaluation of Arterial Repair After the Implantation of Drug-Coated Stent at the Culprit of Acute Coronary Syndrome
}

\author{
Shumpei Kosugi ${ }^{1}$, Masaki Awata ${ }^{2}$, Yasunori Ueda ${ }^{1}$, Haruhiko Abe ${ }^{1}$, Tsuyoshi Mishima ${ }^{1}$, Kazuya Shinouchi ${ }^{1}$, \\ Tatsuhisa Ozaki ${ }^{1}$, Kotaro Takayasu ${ }^{1}$, Yoshinori Iida ${ }^{1}$, Takuya Ohashi ${ }^{1}$, Chieko Toriyama ${ }^{1}$, \\ Masayuki Nakamura ${ }^{1}$, Yasuhiro Ueda ${ }^{1}$, Shun Sasaki ${ }^{1}$, Mikiko Matsumura ${ }^{1}$, \\ Takashi Iehara ${ }^{1}$, Motoo Date ${ }^{1}$, Masaaki Uematsu ${ }^{1}$ and Yukihiro Koretsune ${ }^{1}$
}

\begin{abstract}
BioFreedom is drug-coated stent (DCS) which has polymer-free design. Although it is expected to achieve earlier arterial repair after DCS implantation as compared to the other drug-eluting stents, angioscopic findings have not been described to date. This is the first report of serial angioscopic observation of DCS implanted at acute coronary syndrome (ACS) culprit.

A 75-year-old man was admitted with ACS. Coronary angiogram revealed severe stenosis and thrombus in a large diagonal artery. DCS (BioFreedom $3.0 \times 18 \mathrm{~mm}$ ) was implanted at the culprit of ACS. Coronary angioscopy was performed immediately, one and a half months, and 1 year after stent implantation to evaluate arterial repair after the implantation. Coronary angioscopy showed that uncovered stent struts on the white vessel wall and culprit ruptured yellow plaque with stent struts penetration were observed immediately after stent implantation. At one and a half months, majority of stent struts were not yet covered by neointima and the ruptured yellow plaque remained unhealed with thrombus adhesion. At one year under continued dual antiplatelet therapy, ruptured yellow plaque was covered by white neointima and no thrombus was observed.

Although DCS implanted at ACS culprit was well covered by white neointima without thrombus at 1 year, arterial repair at one and half months after DCS implantation did not appear good yet.
\end{abstract}

KEY WORDS: acute coronary syndrome, coronary angioscopy, drug-coated stent

\section{Introduction}

The BioFreedom drug-coated stent (DCS, Biosensors Int., Singapore) has a polymer-free design with a micro-structured surface coated with Biolimus-A9, which is transferred to the vessel wall over a period of 1 month. A prospective randomized trial showed that the BioFreedom DCS was superior to bare-metal stent (BMS) both in safety- and efficacy-endpoint in high bleeding risk patients with 1-month dual antiplatelet therapy (DAPT), even in acute coronary syndrome (ACS) patients ${ }^{1,2)}$. Therefore, DCS is expected to achieve earlier arterial healing as compared to the other drug-eluting stents (DES). Although neointimal coverage of DCS assessed by optical coherence to-

\footnotetext{
${ }^{1}$ Cardiovascular Division, National Hospital Organization Osaka National Hospital, 2-1-14 Hoenzaka, Chuo-ku, Osaka, 540-0006 Japan, ${ }^{2}$ Cardiovascular Division, Mombetsu General Hospital, Hokkaido, Japan

This paper was presented at the $32^{\text {nd }}$ Annual Meeting of the Japan Coronary Association, 2018, Kumamoto, Japan

E-mail: yellowplaque@gmail.com

Received: September 30, 2019; Accepted: December 20, 2019

J-STAGE Advance Published Date: February 28, 2020

doi: 10.7793/jcad.26.19-00014
}

mography in early phase has been reported to be rapid, serial angioscopic findings of arterial repair after DCS implantation have not been described to date ${ }^{3)}$.

\section{Case report}

A 75-year-old man with hypertension presented to emergency department with 10 days of intermittent rest chest pain. Laboratory evaluation revealed that cardiac troponin I was elevated. Electrocardiogram showed depression of ST segments in lead I, II, aVL, and V3 to V6. Coronary angiogram revealed severe stenosis with a sign of thrombus in the mid large diagonal artery (Fig. 1A) and a total occlusion of distal right coronary artery accompanied by good collateral vessels. We judged the stenosis in the diagonal artery as the culprit of ACS. After the loading of standard doses of aspirin $(200 \mathrm{mg})$ and prasugrel $(20 \mathrm{mg})$, we directly implanted a DCS (BioFreedom 3.0/18 mm) at the culprit (Fig. 1B). Angioscopic observation revealed uncovered struts on the white vessel wall (Fig. 1a) and struts penetration into the ruptured yellow plaque (Fig. 1b, red arrow). The patient received statins and dual DAPT with standard doses of aspirin $(100 \mathrm{mg})$ and prasugrel $(3.75 \mathrm{mg})$. One and a half months later when we 


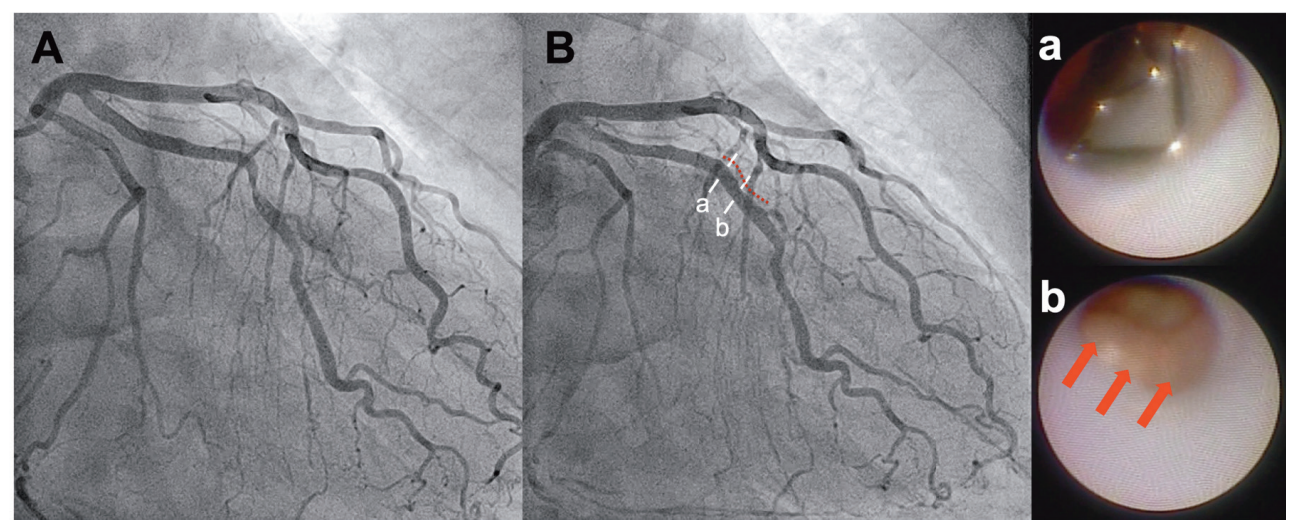

Fig. 1 Angiographic and angioscopic findings immediately after DCS implantation.

Initial coronary angiogram (A) demonstrated severe stenosis with a sign of thrombus in the mid large diagonal artery, which was dilated by DCS implantation (B). Angioscopic images immediately after DCS implantation revealed stent struts on the white vessel wall (a) and ruptured yellow plaque in which stent struts were embedded and invisible (b, red arrow).

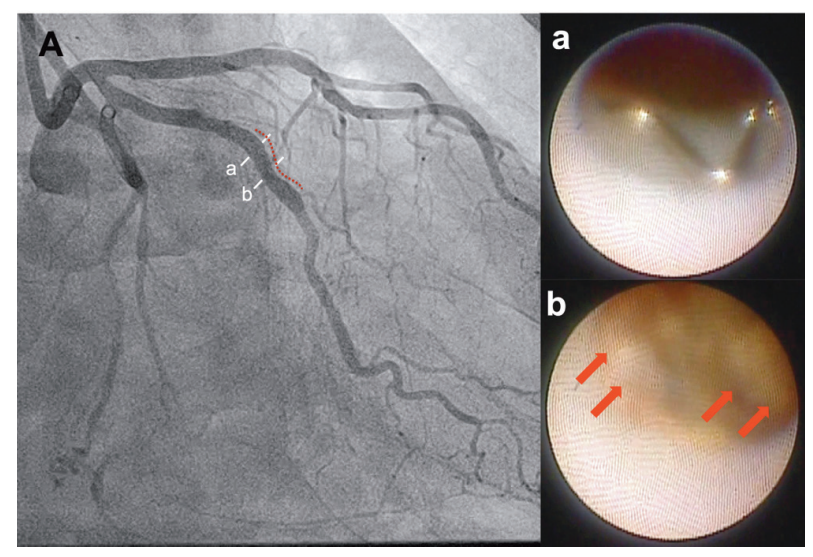

Fig. 2 Angiographic and angioscopic findings at one and a half months follow-up.

Coronary angiogram demonstrated no restenosis (A). Angioscopic findings at one and a half months follow-up revealed still uncovered stent struts (a) and unhealed ruptured yellow plaque with red thrombus (b, red arrow).

performed percutaneous coronary intervention of right coronary artery, angioscopic observation of the stent implanted in the diagonal artery was performed. Although angiogram revealed no restenosis or a sign of thrombus (Fig. 2A), angioscopy demonstrated that majority of stent struts were not yet covered by neointima (Fig. 2a) and the ruptured plaque remained unhealed with thrombus adhesion (Fig. 2b, red arrow). After continuing DAPT for 1 year, follow-up coronary angiogram and angioscopy were performed. Angiogram showed no restenosis (Fig. 3A). Angioscopic observation revealed that the proximal stent struts remained visible but covered by thin neointima (Fig. 3a), and the ruptured yellow plaque was covered by white neointima and no thrombus was observed (Fig. 3b). The majority of struts, covered by neointima, became invisible in the mid and distal portion of

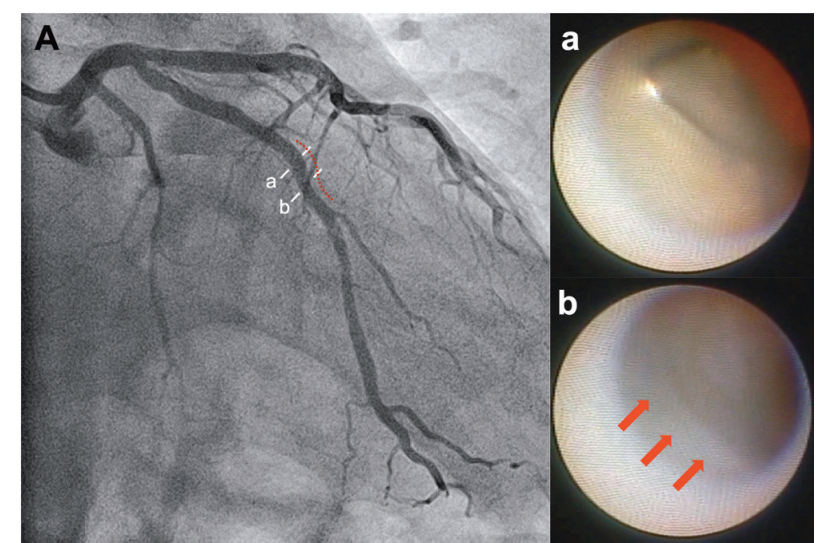

Fig. 3 Angiographic and angioscopic findings at 1-year follow-up.

Coronary angiogram demonstrated no restenosis (A). Angioscopic findings at 1-year follow-up revealed the majority of struts, covered by neointima, became invisible ( $3 \mathrm{~b}$, red arrow) and the proximal struts remained visible but covered by thin neointima (a).

the stent (Fig. 3b, red arrow).

\section{Discussion}

To the best of our knowledge, this is the first report of serial angioscopic observation of DCS implanted at ACS culprit. In this patient, DCS implanted at culprit sites of ACS was sufficiently covered by white neointima and no thrombus adhered at 1 year after the implantation. On the other hand, it had insufficient neointimal coverage with intrastent plaque prolapse at one and a half months after the implantation. The present angioscopic findings suggested that thrombogenicity of target lesion in patient with ACS may remain high at one and a half months after the implantation despite the use of DCS.

In previous autopsy study, a cause of stent thrombosis (ST) in 
patient with acute myocardial infarction was intrastent plaque prolapse $^{4)}$. A stent placed in an artery with a large lipid core with significant plaque prolapse could have a delayed development of endothelialized neointima ${ }^{5)}$. In addition, uncovered strut is also highly associated with occurrence of $\mathrm{ST}^{6}$. Although a randomized comparison between DCS and BMS in high bleeding risk patients receiving 1-month DAPT showed superiority of DCS in safety endpoint, the ST rate of DCS in this trial was higher than previously reported ST rates of the other DES ${ }^{1,7-9}$. We should carefully consider the shortening of DAPT duration, even when DCS is deployed at the culprit of ACS patient.

\section{Conclusion}

DCS implanted at ACS culprit was well covered by white neointima without thrombus at 1 year ; however, it was not covered by neointima with unhealed ruptured plaque at one and half months.

\section{Disclosure statement}

Ueda Y received research grant from Abbott Vascular, Pfizer, Bayer, Daiichi-Sankyo, Astellas, Shionogi, Sanofi, Ono, Nihon Kohden, Amgen Astellas, Actelion, Bristol-Myers Squibb, Medtronic, AstraZeneca, Eisai, Otsuka, and Novartis, and lecture fee from Daiichi-Sankyo, MSD, Nipro-Goodman, Sanofi, Mochida, Takeda, Kowa, Teijin, Astellas, Actelion, Bristol-Myers Squibb, AstraZeneca, Boston Scientific, Sumitomo Dainippon Pharma, Eisai, and Amgen Astellas. All other authors declared no conflict of interest.

\section{References}

1) Urban P, Meredith IT, Abizaid A, et al; LEADERS FREE Investigators: Polymer-free drug-coated coronary stents in patients at high bleeding risk. N Engl J Med 2015; 373: 2038-2047

2) Naber CK, Urban P, Ong PJ, et al; LEADERS FREE Investigators: Biolimus-A9 polymer-free coated stent in high bleeding risk patients with acute coronary syndrome: a Leaders Free ACS sub-study. Eur Heart J 2017; 38: 961-969

3) Lee SWL, Tam FCC, Chan KKW, et al: Establishment of healing profile and neointimal transformation in the new polymer-free biolimus A9-coated coronary stent by longitudinal sequential optical coherence tomography assessments: the EGO-BIOFREEDOM study. EuroIntervention 2018; 14: 780-788

4) Nakazawa G, Finn AV, Joner M, et al: Delayed arterial healing and increased late stent thrombosis at culprit sites after drug-eluting stent placement for acute myocardial infarction patients: an autopsy study. Circulation 2008; 118: 1138-1145

5) Farb A, Burke AP, Kolodgie FD, et al: Pathological mechanisms of fatal late coronary stent thrombosis in humans. Circulation 2003; 108: $1701-1706$

6) Finn AV, Joner M, Nakazawa G, et al: Pathological correlates of late drug-eluting stent thrombosis: strut coverage as a marker of endothelialization. Circulation 2007; 115: 2435-2441

7) Smits PC, Hofma S, Togni M, et al: Abluminal biodegradable polymer biolimus-eluting stent versus durable polymer everolimus-eluting stent (COMPARE II): a randomised, controlled, non-inferiority trial. Lancet 2013; 381: 651-660

8) Kereiakes DJ, Meredith IT, Windecker S, et al: Efficacy and safety of a novel bioabsorbable polymer-coated, everolimus-eluting coronary stent. Circ Cardiovasc Interv 2015; 8: $1-8$

9) Kandzari DE, Koolen JJ, Doros G, et al; BIOFLOW V Investigators: Ultrathin bioresorbable polymer sirolimus-eluting stents versus thin durable polymer everolimus-eluting stents. J Am Coll Cardiol 2018; 72: $3287-3297$ 